Pacific Journal of Mathematics

A THEOREM ON PRIME DIVISORS OF ZERO AND
CHARACTERIZATIONS OF UNMIXED LOCAL DOMAINS 


\title{
A THEOREM ON PRIME DIVISORS OF ZERO AND CHARACTERIZATIONS OF UNMIXED LOCAL DOMAINS
}

\author{
L. J. RATLiFF, JR.
}

Two characterizations of when the prime divisors of zero in a complete local ring are all minimal are given. Then a number of results which characterize an unmixed local domain $R$ of altitude two are proved. Among these are when certain over-rings of $R$ are finite $R$-modules and when $R$ is a subspace of certain Macaulay over-rings. Finally, some characterizations of when an arbitrary local domain is unmixed are given.

1. Introduction. All rings in this article are assumed to be commutative with identity, and the terminology is, in general, the same as that in [6].

In this paper a number of characterizations of when a local domain $R$ is unmixed are proved. The main emphasis is on the case when altitude $R=2(\S \S 3-5)$, but some characterizations for arbitrary local domains are given in $\S 6$. (One reason why characterizations of $R$ being unmixed when altitude $R=2$ are of value is that if $(L, N)$ is a local domain such that altitude $L>2$, then certain monadic transformations $Q$ of $L$ are altitude two local domains, and if $L$ is a subspace of such a $Q$, then $L$ is unmixed if and only if $Q$ is unmixed (4.15). Another reason is that, in answer to a question asked by M. Nagata in 1956 in [5, Problem 1], D. Ferrand and M. Raynaud showed in 1970 in [2, Proposition 3.3] that there exist quasi-unmixed local domains of altitude two which aren't unmixed. This is considered somewhat more fully in $\S 5$.) A number of these characterizations in altitude two are due to a more general theorem concerning when the prime divisors of zero in a complete local ring are all minimal, and others have to do with certain Macaulay overrings of $R$.

A brief summary of the results in this paper will now be given. Throughout the remainder of this introduction $(R, M)$ is a local domain such that altitude $R=2$.

The main result in $\S 2$ shows that if $(L, N)$ is a complete local ring and altitude $L \geqq 2$, then every prime divisor of zero in $L$ is minimal and has depth $>1$ if and only if there exist a regular element $b \in N$ and $n \geqq 1$ such that $(b L)^{(n)} \subseteq b L$, where $(b L)^{(n)}$ is the intersection of the height one primary components of $b^{n} L$ (2.2).

In $\S 3$, using (2.2), six characterizations of when $R$ (altitude two) is unmixed are given in (3.4) and (3.6). Among these are: $(b R)^{(n)} \subseteq$ 
$b R$, for some $0 \neq b \in M$ and $n \geqq 1$; and, there exist a system of parameters $b, c$ in $R$ and $n \geqq 1$ such that $b^{k} R: c^{n} R=(b R)^{(k)}$, for all $k \geqq 1$.

Section 4 contains some characterizations of $R$ being unmixed in terms of $R$ being a subspace of rings of the form $A_{P}$, where $b, c$ is a system of parameters in $R$ and $P$ is a prime ideal in $A=R[b / c]$ such that $M A \subseteq P$. Specifically, $R$ is unmixed if and only if $R$ is a subspace of one such ring which is Macaulay if and only if $R$ is a subspace of all such rings (4.9) and (4.10). Some further results on when $A$ is locally Macaulay or when $A_{(M, b / c)}$ is Macaulay are given in (4.1), (4.5), (4.7), and (4.8).

Section 5 contains characterizations of $R$ being unmixed or quasi-unmixed in terms of $R^{(1)}$ and $R^{\prime}$ (5.1). (5.2) contains some supplemental information on the relationships between the rings $R$, $R^{(1)}$, and $R^{\prime}$.

In $\S 6$ a sufficient condition for a local ring $L$ to be unmixed is given in (6.1). Then, using (6.1), a number of characterizations of when $L$ is unmixed and of when $L / p$ is unmixed, for all $p \in$ Spec $L$, are given in (6.3)-(6.9).

2. A theorem on prime divisors of zero. The main result in this section gives two characterizations of when every prime divisor of zero in a complete local ring is minimal and has depth $>1$ (2.2). To prove (2.2) we need the following definition.

Definition 2.1. Let $b$ be a regular element in a Noetherian ring $A$. Then $(b A)^{(n)}=b^{n} A_{S} \cap A$, where $S=A-\cup\{p \in \operatorname{Spec} A ; p$ is a height one prime divisor of $b A\}$.

Thus, by the definition, $(b A)^{(n)}$ is the isolated component of $b^{n} A$ determined by the minimal prime divisors of $b^{n} A$.

In (2.2) we restrict attention to local rings of altitude $\geqq 2$, since if $(R, M)$ is a local ring and altitude $R=1$, then every prime divisor of zero in $R$ is minimal if and only if $M$ contains regular elements, and if altitude $R=0$, then every prime divisor of zero in $R$ is minimal. I am indebted to S. McAdam for showing that $(2.2 .1) \Rightarrow$ (2.2.2) for all local rings (instead of just for complete local rings).

THEOREM 2.2. Let $(R, M)$ be a local ring such that altitude $R \geqq 2$, and consider the following statements:

(2.2.1) Each prime divisor $z$ of zero in $R$ is such that height $z=0$ and depth $z>1$.

(2.2.2) There exists a regular element $b \in M$ such that $\bigcap_{n}(b R)^{(n)}=(0)$.

(2.2.3) There exist a regular element $b \in M$ and $n \geqq 1$ such that $(b R)^{(n)} \subseteq b R$. 
Then $(2.2 .3) \Rightarrow(2.2 .2) \Leftrightarrow(2.2 .1)$. Moreover, if $R$ is complete, then all three statements are equivalent.

Proof. It will be shown in (3.3.2) below that if $(b R)^{(n)} \subseteq b R$, then $(b R)^{(n+i)} \subseteq b^{i+1} R$, for all $i \geqq 0$, so $(2.2 .3) \Rightarrow(2.2 .2)$.

Assume that (2.2.2) holds, and let $\mathscr{P}$ be the set of height one prime divisors of $b R$. Then $(0)=\bigcap_{n}(b R)^{(n)}=\bigcap\left\{\bigcap_{n} b^{n} R_{p}\right) \cap R$; $p \in \mathscr{P}\}=\bigcap\left\{(0) R_{p} \cap R ; p \in \mathscr{P}\right\}$. Therefore, if $z$ is a prime divisor of (0) in $R$, then there exists $p \in \mathscr{P}$ such that $z \subset p$, so height $z=0$ and depth $z>1$ (since altitude $R \geqq 2$ ), so (2.2.1) holds.

(2.2.1) $\Rightarrow(2.2 .2) \quad$ Let $U=\{q \in$ Spec $R$; there exists a prime divisor $z$ of zero such that $z \subset q$ and height $q / z=1<$ height $q$. Then, by hypothesis, $U$ is a finite set (possibly empty) [4, Theorem 1] and $M \notin U$. Therefore let $b$ be a regular element in $M$ such that $b \notin \bigcup\{q ; q \in U\}$. Let $\mathscr{P}$ be the set of height one prime divisors of $b R$, and assume it is known that for each prime divisor $z$ of (0), there exists $p \in \mathscr{P}$ such that $z \subset p$. Then $(0)=$ (by assumption) $\left.\bigcap\left\{(0) R_{p} \cap R ; p \in \mathscr{P}\right\}=\bigcap\left\{\bigcap_{n} b^{n} R_{p}\right) \cap R ; p \in \mathscr{P}\right\}=\bigcap_{n}(b R)^{(n)}$. Therefore it remains to show that each prime divisor of zero is contained in some $p \in \mathscr{P}$.

For this, let $z$ be a prime divisor of zero and consider $R / z$. Then the Principal Ideal Theorem implies that $M / z$ is the union of $\{Q / z ; z \subset Q \in \operatorname{Spec} R$ and height $Q / z=1\}$. Therefore there exists $Q \in$ Spec $R$ such that $(z, b) R \subseteq Q$ and height $Q / z=1$. By the choice of $b$, height $Q=1$, so $Q \in \mathscr{P}$, and so (2.2.2) holds. In fact, it has been shown that:

(2.2.4) For each regular element $b \in M, \notin \bigcup\{q: q \in U\}$, $\bigcap_{n}(b R)^{(n)}=(0)$.

Finally, assume that $R$ is complete and that (2.2.1) holds. Let $U$ and $b$ be as in the proof that $(2.2 .1) \Rightarrow(2.2 .2)$, let $P$ be a prime divisor of $b R$, and let $m$ be a positive integer. Then to show that (2.2.3) holds it suffices to show that there exists $k \geqq 1$ such that $(b R)^{(k)} \cong P^{(m)}=P^{m} R_{P} \cap R$ (since $b R$ has a primary decomposition and primary ideals contain large symbolic powers of their prime divisor).

For this, it may clearly be assumed that height $P>1$. Let $(S, N)$ be the completion of $\left(R_{P}, P R_{P}\right)$. Then, since $R$ is complete, for each prime divisor $z$ of $(0)$ in $R_{P}$, the completion $\left(R_{P} / z\right)^{*}$ of $R_{P} / z$ is analytically unramified and unmixed [5, Proposition 4]. Therefore, all prime divisors of $(0)$ in $S$ are minimal, for if $w$ is a prime divisor of zero in $S$, then $w$ is a prime divisor of $z S$, sor some prime divisor $z$ of $(0)$ in $R_{P}$, so height $w=0$ (since $S / z S \cong\left(R_{P} / z\right)^{*}$ is analytically unramified). Suppose there exists a prime divisor $z^{\prime}$ of (0) in $R$ such that $z^{\prime} \subset P$ and height $P / z^{\prime}=1$. Then height $P=1$, since $b \notin \bigcup\{q ; q \in U\}$; contradiction. Therefore every prime 
divisor of (0) in $R_{P}$ has depth $>1$, so every prime divisor of (0) in $S$ has depth $>1$ (since the $\left(R_{P} / z\right)^{*}$ are unmixed). Thus $S$ satisfies the conditions on $R$ in (2.2.1). Now, if it can be shown that $b \notin \bigcup\left\{q^{\prime} ; q^{\prime} \in U^{\prime}\right\}$, where $U^{\prime}=\left\{q^{\prime} \in\right.$ Spec $S$; there exists a prime divisor $w$ of zero in $S$ such that $w \subset q^{\prime}$ and height $q^{\prime} / w=1<$ height $\left.q^{\prime}\right\}$, then $\bigcap_{n}(b S)^{(n)}=(0)$ (by $(2.2 .4)$ applied to $S$ ). It then follows that, given $m \geqq 1$, there exists $k$ such that $(b S)^{(k)} \cong N^{m}[6,(30.1)]$. So, since height one prime divisors of $b S$ contract to height one prime divisors of $b R_{P},\left(b R_{P}\right)^{(k)} \subseteq(b S)^{(k)} \cap R_{P} \subseteq N^{m} \cap R_{P}=P^{m} R_{P}$, hence $(b R)^{(k)} \subseteq P^{(m)}$. Thus it remains to show that $b \notin \bigcup\left\{q^{\prime} ; q^{\prime} \in U^{\prime}\right\}$.

For this, suppose $b \in \bigcup\left\{q^{\prime} ; q^{\prime} \in U^{\prime}\right\}$ and let $p \in U^{\prime}$ such that $b \in p$. Then there exists a (minimal) prime divisor $w$ of (0) in $S$ such that $(w, b) S \subseteq p$ and height $p / w=1<$ height $p$. Since height $p / w=1$, $p$ is a prime divisor of $b S$ (by [17, Lemma 1, p. 394] applied to $S_{p}$ ), so $q=p \cap R_{P}$ is a prime divisor of $b R_{P}$, by [6, (18.11)]. Since height $p>1$, there exists another prime divisor $w^{\prime}$ of $(0)$ in $S$ such that $w^{\prime} \subset p$ and depth $w^{\prime}>\operatorname{depth} w$ (since $S$, being complete, is catenary). Therefore $z^{\prime}=w^{\prime} \cap R_{P} \neq w \cap R_{P}=z$ (since $S / z S$ is unmixed). Thus $z^{\prime}$ and $z$ are contained in $q$ and depth $z^{\prime}=\operatorname{depth} w^{\prime}>\operatorname{depth} w=$ depth $z$, so height $q>1$ (since $R_{P}$ is catenary, (since $R$ is catenary)). Also, height $p / z S=1$ and $b+z S \in p / z S$, so height $\left((p / z S) \cap\left(R_{P} / z\right)\right)=1$, hence height $q / z=1$. Therefore height $(q \cap R) /(z \cap R)=1$ and height $q \cap R>1$, so $b \in q \cap R \in U$; contradiction. Therefore $b \notin \bigcup\left\{q^{\prime}\right.$; $\left.q^{\prime} \in U^{\prime}\right\}$, so $(2.2 .3)$ holds.

REMARK 2.3. The proof that $(2.2 .1) \Rightarrow(2.2 .3)$ shows that for each regular element $b \in M$ such that $b \notin \bigcup\{q ; q \in U\}$, there exists $n \geqq 1$ such that $(b R)^{(n)} \subseteq b R$ (assuming that $R$ is complete).

It should be noted that if altitude $R=2$ in (2.2), then the proof that $(2.2 .1) \Rightarrow(2.2 .3)$ follows immediately from [6, (30.1)] (and the fact that $(2.2 .1) \Rightarrow(2.2 .2)$ ), since then $S=R$ (with $S$ as in the proof that $(2.2 .1) \Rightarrow(2.2 .3))$. It should also be mentioned that a somewhat different proof that $(2.2 .2) \Rightarrow(2.2 .1)$ in an arbitrary Noetherian ring such that each maximal ideal has height $>1$ is given in [15, Lemma 4.11].

Corollary 2.4. Let $(R, M)$ be a quasi-unmixed local domain such that altitude $R \geqq 2$, and let $R^{*}$ be the completion of $R$. Then the following statements are equivalent:

(2.4.1) $R$ is unmixed.

(2.4.2) There exists $0 \neq b \in M$ such that $\bigcap_{n}\left(b R^{*}\right)^{(n)}=(0)$.

(2.4.3) For each $0 \neq b \in M$, there exists $n \geqq 1$ such that $\left(b R^{*}\right)^{(n)} \subseteq b R^{*}$.

Proof. If (2.4.1) holds, then, since the set $U$ (for $R^{*}$ ) of the 
proof that $(2.2 .1) \Rightarrow(2.2 .3)$ is empty, $(2.4 .3)$ holds by (2.3).

(2.4.3) $\Rightarrow$ (2.4.2), by (3.3.2) below.

If (2.4.2) holds, then the prime divisors of zero in $R^{*}$ are all minimal (2.2), so, since $R$ is quasi-unmixed (by hypothesis), (2.4.1) holds.

It will be shown in (3.4) that a much nicer result than (2.4) holds when altitude $R=2$.

3. Some characterizations of unmixed local domains of altitude two. It will be shown in this section that (2.2) can be used to give a number of characterizations of an unmixed local domain of altitude two. In fact, six such characterizations are given in (3.4) and (3.6).

Most of the results in this section can be extended to altitude two local rings (with nonzero divisors of zero), but we shall restrict attention to the domain case. On the other hand, only a small part of the results extend to local domains of altitude $>2$.

To prove (3.4) and (3.6) we need the following definitions.

Definitions 3.1. Let $A$ be a Noetherian domain, and let $b$ be a nonzero element in $A$.

(3.1.1) $A^{(1)}=\bigcap\left\{A_{p} ; p\right.$ is a height one prime ideal in $\left.A\right\}$.

(3.1.2) $\mathscr{T}(b A)=A[1 / b] \cap A^{(1)}$.

(3.1.3) An ideal $I$ in $A$ is pure height one in case every prime divisor of $I$ has height one.

Quite a few results concerning one or another of these concepts are given in [3, $\S 5.10$ and 5.11], [9, §5], [10, pp. 218-219], and [15, Chapter 2] (among other possible references). The following remark lists the known facts which are needed for the remainder of this paper.

REMARK 3.2. Let $b$ a nonzero non-unit in a Noetherian domain. Then the following statements hold:

3.2.1. [10, Corollary 2.19(1)]. There exists a one-to-one correspondence between the height one prime ideals $p$ in $A$ and the height one prime ideals $q$ in $A^{(1)}: p$ and $q$ correspond if and only if $A_{p}=A_{q}^{(1)}$.

3.2.2. [3, (5.10.17) (1)]. Each proper nonzero principal ideal in $A^{(1)}$ is pure height one.

3.2.3. [9, Lemma 5.15(3)] and [15, Lemma 2.11(3)]. $\mathscr{T}(b A)$ is the set of elements which, for all large $k$, can be written in the form $c / b^{k}$ with $c \in(b A)^{(k)}$.

3.2.4. [9, Lemma 5.15(4)] and [15, Lemma 2.17(1)]. $\quad b^{k} \mathscr{T}(b A) \cap$ 
$A=(b A)^{(k)}$, for all $k \geqq 1$.

3.2.5. [9, Lemma 5.15(10)]. $\mathscr{T}(b A)$ is a finite $A$-algebra if and only if $b^{n} \mathscr{T}(b A) \subseteq A$, for some $n \geqq 1$.

3.2.6. [9, Lemma 5.15(4)] and [15, Lemma 2.11(5)]. $b \mathscr{T}(b A)$ is pure height one.

The following lemma will serve to shorten the proofs of 3.4 and 3.6 .

LEMMA 3.3. Let $b$ be a regular nonunit in a Noetherian ring A. Then the following statements hold:

3.3.1. $(b A)^{(n)}: b^{i} A=(b A)^{(n-i)}$, for all $n \geqq i \geqq 0$.

3.3.2. If $(A b)^{(n)} \leqq b A$, for some $n \geqq 1$, then $(b A)^{(m)}=b^{m-n+1}(b A)^{(n-1)}$, for all $m \geqq n$.

Proof. 3.3.1. $(b A)^{(n)}: b^{i} A$ and $(b A)^{(n-i)}$ are pure height one, they have the same height one prime divisors as $b A$, and $\left((b A)^{(n)}: b^{i} A\right) A_{p}=$ $b^{n-i} A_{p}=\left((b A)^{(n-i)}\right) A_{p}$, for each height one prime divisor $p$ of $b A$, hence they are equal.

3.3.2. Let $m \geqq n$, so $(b A)^{(m)}=(b A)^{(m)} \cap b A=b\left((b A)^{(m)}: b A\right)=$ 3.3.1 $b(b A)^{(m-1)}$, so the conclusion follows by induction on $m-n$.

The first main result in this section gives four characterizations of an unmixed local domain of altitude two. Of these, it is already known that 3.4.1 $\Leftrightarrow 3.4 .5$, by [9, Theorem 3.5] (which is a sharpened version of $[3,7.2 .5])$.

THEOREM 3.4. The following statements are equivalent for a local domain $(R, M)$ such that altitude $R=2$ :

3.4.1. $R$ is unmixed.

3.4.2. $(b R)^{(n+i)}=b^{i}(b R)^{(n)}$, for each $0 \neq b \in M$, for some $n$ depending on $b$, and for all $i \geqq 0$.

3.4.3. $(b R)^{(n)} \subseteq b R$, for some $0 \neq b \in M$ and for some $n$.

3.4.4. $\mathscr{T}(b R)$ is a finite $R$-algebra, for some $0 \neq b \in M$.

3.4.5. $R^{(1)}$ is a finite $R$-algebra.

Proof. Assume that 3.4.1 holds and let $0 \neq b \in M$. Then, with $R^{*}$ the completion of $R$, the set $\left\{q \in\right.$ Spec $R^{*}$; there exists a prime divisor $z$ of zero such that $z \subset q$ and height $q / z=1<$ height $q$ \} is empty. Thus there exists $n$ (depending on $b$ ) such that $\left(b R^{*}\right)^{(n)} \subseteq b R^{*}$ 2.3. Therefore $(b R)^{(n)} \subseteq\left(b R^{*}\right)^{(n)} \cap R \cong b R^{*} \cap R=b R$, so 3.4 .2 holds by 3.3.2.

It is clear that $3.4 .2 \Rightarrow 3.4 .3$.

Assume that 3.4 .3 holds and let $x \in \mathscr{T}(b R)$. Then, by 3.2.3, $x=c / b^{k}$, for some $c \in(b R)^{(k)}$, and it may be assumed that $k \geqq n$. 
Therefore $c \in b^{k} \mathscr{T}(b R) \cap R=3.2 .4 \quad(b R)^{(k)} \subseteq(b R)^{(n)} \subseteq b R$, so $c=c_{1} b$, for some $c_{1} \in R$. Thus $x=c_{1} / b^{k-1}$, so it follows that $x=c^{\prime} / b^{n-1}$, for some $c^{\prime} \in R$. Therefore $b^{n-1} \mathscr{T}(b R) \subseteq R$, hence $\mathscr{T}(b R)$ is a finite $R$ algebra, and so 3.4.4 holds.

$R[1 / b]=\bigcap\left\{R[1 / b]_{p} ;(0) \neq p \in \operatorname{Spec} R[1 / b]\right\}=\bigcap\left\{R_{q} ; b \notin q \in \operatorname{Spec} R\right\}$, since altitude $R[1 / b]=1$, so it follows from the definitions that $\mathscr{T}(b R)=R^{(1)}$. Therefore $3.4 .4 \Rightarrow 3.4 .5$.

Finally, assume that 3.4.5 holds. Then $R^{(1)}$ is Noetherian and $R^{(1)} \cong R^{\prime}=$ the integral closure of $R$, hence altitude $R^{(1)}=$ altitude $R=2$. Also, each nonzero proper principal ideal is pure height one 3.2.2, and height one prime ideals in $R^{(1)}$ contract to height one prime ideals in $R$ 3.2.1. Therefore $R^{(1)}$ is Macaulay and height $M^{\prime}=2$, for each maximal ideal $M^{\prime}$ in $R^{(1)}$ (by 3.2 .1 and since maximal ideals in $R^{(1)}$ lie over maximal ideals in $R$, by integral dependence). Thus $R^{(1)}$ is an unmixed semi-local domain and is a finite $R$-algebra, so $R$ is unmixed (since the completions of $R^{(1)}$ and $R$ have the same total quotient ring).

For future reference we note the following fact which was proved in showing $3.4 .4 \Rightarrow 3.4 .5$.

REMARK 3.5. With the notation of $3.4, R^{(1)}=\mathscr{T}(b R)$, for each $0 \neq b \in M$.

With $(R, M)$ as in 3.4 , let $b, c$ be a system of parameters in $R$. Then, for some $n \geqq 1, b R: c^{n} R=(b R)^{(1)}$. (Choose $n$ such that either $c^{n}$ is in an $M$-primary component of $b R$, or $n=1$ if $b R: M=b R$.) Now, for all $k \geqq 1, b^{k}, c$ is also a system of parameters. Thus for some $n(k)$ we have $b^{k} R: c^{n(k)} \mathrm{R}=\left(b^{k} R\right)^{(1)}=(b R)^{(k)}$. Here $n(k)$ depends on $k$. In the following theorem it is shown that $R$ is unmixed if and only if $n(k)$ can be chosen independent of $k$.

Theorem 3.6. Let $(R, M)$ be as in 3.4. Then the following statements are equivalent:

3.6.1. $R$ is unmixed.

3.6.2. For each system of parameters $b, c$ in $R, b^{k} R: c^{n} R=(b R)^{(k)}$, for all $k \geqq 1$ and for all large $n$.

3.6.3. There exist a system of parameters $b, c$ in $R$ and $n \geqq 1$ such that $b^{k} R: c^{n} R=(b R)^{(k)}$, for all large $k$.

Proof. Assume that 3.6.1 holds and let $b, c$ be a system of parameters in $R$. Then there exists $h \geqq 1$ such that $(b R)^{(k)}=$ $b^{k-h}(b R)^{(h)}$, for all $k \geqq h 3.4$. If $M$ isn't a prime divisor of $b R$, then clearly 3.6.2 holds, so assume that $M$ is a prime divisor of $b R$. For $i=1, \cdots, h+1$, let $Q_{i}$ be a $M$-primary component of $b^{i} R$, and let 
$n$ such that $c^{n} \in \bigcap_{1}^{h+1} Q_{i}$. Then $b^{i} R: c^{n} R=(b R)^{(i)}$, for $i=1, \cdots, h$, and $b^{h+1} R: c^{n} R=(b R)^{(h+1)}=b(b R)^{(h)} \leqq b R$. Therefore $b^{h+i} R: c^{n} R=$ $\left(b^{h+i} R: c^{n} R\right) \cap b R=b\left(b^{h+i} R: c^{n} b R\right)=b\left(b^{h+i-1} R: c^{n} R\right)=$ (by induction) $b\left(b^{i-1}(b R)^{(h)}\right)=b^{i}(b R)^{(h)}=(b R)^{(h+i)}$. 3.6.2 clearly follows from this.

It is clear that $3.6 .2 \Rightarrow 3.6 .3$.

Finally, assume that 3.6 .3 holds. Now there exists $r \geqq 0$ such that $b^{r+i} R: c^{n} R=b^{i}\left(b^{r} R: c^{n} R\right)$, for all $i \geqq 0[6$, Ex. 3, p. 12]. Therefore, for $i$ large, $(b R)^{(r+i)}=b^{r+i} R: c^{n} R \subseteq b^{i} R$, so

$$
(b R)^{(r+1)}=3.3 .1(b R)^{(r+i)}: b^{i-1} R \cong b^{i} R: b^{i-1} R=b R,
$$

hence 3.6.1 holds by 3.4.

4. Macaulay over-rings of an altitude two local domain. In this section we first give some necessary and sufficient conditions for $A=R[b / c]$ to be locally Macaulay and for $A_{(M, b / c)}$ to be Macaulay where $b, c$ is a system of parameters in an altitude two local domain $(R, M) 4.1,4.5,4.7$, and 4.8. Then we use these results to characterize when $R$ is unmixed in 4.10-4.14. Finally, we show in 4.15 that such characterizations can be used to obtain information on when local domains of altitude $>2$ are unmixed.

We begin with the following result.

Proposition 4.1. Let $b, c$ be a system of parameters in a local domain $(R, M)$ such that altitude $R=2$. Then $A=R[b / c]$ is locally Macaulay if and only if $c A$ is MA-primary.

Proof. $M A$ is a height one depth one prime ideal [9, Lemma 4.3], so $M A$ is a prime divisor of $c A$. Assume $c A$ isn't $M A$-primary, and let $p \neq M A$ be a prime divisor of $c A$. Then $(b, c) R \cong p \cap R$, so $p \cap R=M$, and so $M A \subseteq p$, hence $p$ is an imbedded prime divisor of $c A$.

Now, if $A$ is locally Macaulay, then, in particular, $A_{N}$ is Macaulay, for all prime ideals $N$ in $A$ such that $M A \subset N$. Therefore $N$ isn't a prime divisor of $c A$, so $c A$ is $M A$-primary (by the preceding paragraph).

Conversely, if $c A$ is $M A$-primary, then, for each height two maximal ideal $N$ in $A, M A \subset N$ and $c A_{N}$ is $M A_{N}$-primary, so $A_{N}$ is Macaulay. It readily follows from this that $A$ is locally Macaulay, since altitude $A=2$.

The following remark gives some information related to 4.1. 
REMARK 4.2. Let the notation be as in 4.1 , and let $x=b / c$. Then the following statements hold:

4.2.1. $\operatorname{Rad} c A=M A$, even if $A$ isn't locally Macaulay.

4.2.2. $A_{N}$ is Macaulay, for all but a finite number of maximal ideals $N$ in $A$.

4.2.3. $A_{(M, x) A}$ is Macaulay if and only if $x A$ is pure height one.

Proof. 4.2.1 was proved in the first paragraph of the proof of 4.1.

4.2.2. If $A_{N}$ isn't Macaulay, then height $N=2$ (so $M A \subset N$ ) and $N A_{N}$ is a prime divisor of the nonzero principal ideals contained in $N A_{N}$, hence $N$ is a prime divisor of $c A$. The conclusion follows, since $c A$ has only finitely many prime divisors.

4.2.3. $A / x A=R /(x A \cap R)$ is a local ring, so every prime divisor of $x A$ is contained in $N=(M, x) A$. Also, $A_{N}$ is Macaulay if and only if $x A_{N}$ is pure height one, so $A_{N}$ is Macaulay if and only if $x A$ is pure height one.

To derive some further information on when $A_{(., y, b / c)}$ is Macaulay, the following definitions and lemma are needed.

Definition 4.3. Let $I$ be an ideal in a Noetherian domain $A$, and let $b \in I$. Then $b$ is superficial for $I$ in case $I^{n+1}: b A=I^{n}$, for all large $n . \quad b$ is strongly superficial for $I$ in case $I^{n+1}: b A=I^{n}$, for all $n \geqq 1$.

By $[6,3.12]$, this definition of superficial element is equivalent to the usual definition (see $[6$, p. 72$]$ or $[17$, p. 285]).

Strongly superficial elements were defined and studied in [16].

The following lemma will be useful in what follows. I am indebted to the referee for suggesting 4.4 .2 and for simplifying my original proofs of 4.5 and 4.7 .

Lemma 4.4. Let $b, c$ be nonunits in a Noetherian domain $A$. Then the following statements hold:

4.4.1. $b$ is superficial (respectively, strongly superficial) for $(b, c) A$ if and only if $c^{n+1} A: b A \subseteq(b, c)^{n} A$, for all large $n$ (respectively, for all $n \geqq 1$ ).

4.4.2. $b$ is superficial (respectively, strongly superficial) for $(b, c) A$ if and only if $b(b, c)^{n} A: c^{n+1} A=b A: c^{n+1} A$, for all large $n$ (respectively, for all $n \geqq 1$ ).

Proof. $(b, c)^{n+1} A: b A=\left(c^{n+1}, b(b, c)^{n}\right) A: b A=\left(c^{n+1} A: b A,(b, c)^{n}\right) A$. Both conclusions in 4.4.1 readily follow from this. 
To prove 4.4.2, fix $n \geqq 1$. Then it suffices to show that $(b, c)^{n+1} A: b A=(b, c)^{n} A$ if and only if $b(b, c)^{n} A: c^{n+1} A=b A: c^{n+1} A$, and the proof of this is straightforward.

Proposition 4.5. Let the notation be as in 4.1. Then $b$ is superficial for $(b, c) R$ if and only if $D=A_{(M, b / c)}$ is Macaulay.

Proof. Let $x=b / c$, and let $I_{n}=b(b, c)^{n} R: c^{n+1} R$, so $I_{n} \subseteq I_{n+1}$ and a straightforward computation shows that $x A \cap R=I_{n}$, for all large $n$. Now, $b$ is superficial for $(b, c) A$ if and only if $I_{n}=b A: c^{n+1} A$, for all large $n 4.4 .2$, if and only if $I_{n}=(b R)^{(1)}$, for all large $n$, if and only if $I_{n}$ is pure height one, for all large $n$, if and only if $x A \cap R$ is pure height one if and only if $x A$ is pure height one (since $A / x A=R /(x A \cap R)$ ) if and only if $D$ is Macaulay.

It is known that if $R / M$ is infinite, then there exists a superficial element, say $d$, for $(b, c) R$ [17, Remark 2, p. 287]. Then it is easily seen that $d \notin(b, c) M$, so either $(b, c) R=(d, b) R$ or $(b, c) R=$ $(d, c) R$, hence either $R[d / b]_{(M, d / b)}$ or $R[d / c]_{(M, d / c)}$ is Macaulay, by 4.5.

Also, it is known [7, Corollary 2.2 and Lemma 2.1] that if $R$ is Macaulay and $b, c$ is a system of parameters in $R$, then $D=R[b / c]_{(M, b / c)}$ is Macaulay and $K=\operatorname{Ker}(R[X] \rightarrow R[b / c])=(c X-b) R[X]$. It will be seen in 4.7 that a weaker condition than being a $R$-sequence implies that $D$ is Macaulay and $K$ has a similar set of generators, even if $R$ is not Macaulay. For this we need the following definition.

DEFINITION 4.6. Let $b, c$ be nonzero elements in an integral domain $A$. Then $K=\operatorname{Ker}(R[X] \rightarrow R[b / c])$ has a linear base in case $K$ is generated by $\{e X-d ; b e=c d\}$.

A number of results on linear bases are given in [11].

Proposition 4.7. With the notation of 4.1, if $b R: c R=(b R)^{(1)}$, then $b$ is strongly superficial for $(b, c) R$ and $K$ has a linear base. Therefore $R[b / c]_{(M, b / c)}$ is Macaulay.

Proof. If $b R: c R=(b R)^{(1)}$, then clearly $b R: c R=b R: c^{n} R$, for all $n \geqq 1$, so $K$ has a linear base [11, Corollary 7]. To see that $b$ is strongly superficial for $(b, c) R$, let $r \in c^{n+1} R: b R$, so $r b=c^{n+1} s$, for some $s \in R$. Then $s \in b R: c^{n+1} R=b R: c R$, so there exists $t \in R$ such that $r b=s c^{n+1}=t b c^{n}$. Therefore $r \in c^{n} R \subseteq(b, c)^{n} R$, hence $b$ is strongly superficial for $(b, c) R$ 4.4.1. The last statement follows from this and 4.5.

It can be shown that the following statements are equivalent: (i) $b$ is strongly superficial for $(b, c) R$ and $K$ has a linear base; (ii) $b$ 
is superficial for $(b, c) R$ and $K$ has a linear base; (iii) $c^{2} R: b R=$ $c(c R: b R)$; and, (iv) $b R: c R=(b R)^{(1)}$. (The proof is fairly straightforward (using 4.7), so will be omitted.)

One further result on Macaulay over-rings of $R$ will be given. Namely, we will characterize when $R[b / c]$ and $R[c / b]$ are simultaneously locally Macaulay. For this, recall that if $I$ is an ideal in a ring $A$, then the Rees ring $\mathscr{R}=\mathscr{R}(A, I)$ of $A$ with respect to $I$ is the graded subring $\mathscr{R}=A[t I, u]$ of $A[t, u]$, where $t$ is an indeterminate and $u=1 / t$.

Proposition 4.8. With the notation of 4.1, the following statements are equivalent:

4.8.1. $u \mathscr{R}(R,(b, c) R)$ has no height two prime divisors.

4.8.2. For each $0 \neq d \in(b, c) R, R[b / d, c / d]$ is locally Macaulay.

4.8.3. $R[b / c]$ and $R[c / b]$ are locally Macaulay.

Proof. Assume that 4.8.1 holds and let $0 \neq d \in(b, c) R$. Let $A=R[b / d, c / d]$, and let $\mathscr{S}=A[t d, 1 / t d]$, so $\mathscr{S}=\mathscr{R}[1 / t d]$, where $\mathscr{R}=\mathscr{R}(R,(b, c) R)$. Also, $\mathscr{S}$ is a quotient ring of a transcendental extension ring of $A$, and $d \mathscr{S}=u \mathscr{S}$. Let $P$ be a prime ideal in $A$. If height $P=1$, then clearly $A_{P}$ is Macaulay, so assume that height $P=2$ (note that altitude $A \leqq 2$ ). Then $M=P \cap R$, so $d \in P$. Let $P^{*}=P \mathscr{S} \cap \mathscr{R}$. Then height $P^{*}=2$ and $u \in P^{*}$, so $P^{*}$ isn't a prime divisor of $u \mathscr{R}$ (by hypothesis), and so $P^{*} \mathscr{S}$ isn't a prime divisor of $u \mathscr{S}=d \mathscr{S}$, whence $P$ isn't a prime divisor of $d A$, and hence $A_{P}$ is Macaulay.

It is clear that $4.8 .2 \Rightarrow 4.8 .3$.

Finally, assume that 4.8.3 holds, and suppose that $Q$ is a height two prime divisor of $u \mathscr{R}$. Then either $t b$ or $t c$ isn't in $Q$, for otherwise $b=u t b$ and $c=u t c$ are in $Q$ so $Q \cap R$ is $M$-primary (since height $(b, c) R=2)$, hence $(u, M, t b, t c) \mathscr{R} \subseteq Q$, and so height $Q=3$ [9, Remark 3.7]. Say $t c \notin Q$. Then $P=Q \mathscr{R}[1 / t c] \cap R[b / c]$ is a height two prime ideal which is a prime divisor of $c R[b / c]$ (much as in the first paragraph of this proof), so $R[b / c]_{P}$ isn't Macaulay; contradiction. Therefore $u \mathscr{R}$ has no height two prime divisors, so 4.8 .1 holds.

We now return to the consideration of unmixed local domains.

Proposition 4.9. Let $(R, M)$ be an unmixed local domain such that altitude $R=2$, and let $b, c$ be a system of parameters in $R$. Then, for each prime ideal $P$ in $A=R[b / c]$ such that $M A \subseteq P, R$ is a subspace of $A_{P}$.

Proof. This is a special case of $[9,4.1(2)]$. 
Using 4.9 and the preceding results on Macaulay over-rings of $R$, we can now prove a number of characterizations of when $R$ is unmixed.

Corollary 4.10. With the notation of 4.1, let $P$ be a prime ideal in $A$ such that $M A \subseteq P$ and $A_{P}$ is Macaulay. Then $R$ is a subspace of $A_{P}$ if and olny if $R$ is unmixed.

Proof. If $R$ is a subspace of $A_{P}$, then $R$ is unmixed by [9, Lemma 4.5(2)]. The converse follows from 4.9 .

COROLLARY 4.11. With the notation of 4.1, assume that $c A$ is MA-primary. Then there exists a prime ideal $P$ in $A$ such that $M A \subseteq P$ and $R$ is a subspace of $A_{P}$ if and only if $R$ is unmixed.

Proof. A is locally Macaulay 4.1, so the conclusion follows from 4.10 .

COROLlaRY 4.12. With the notation of 4.1, assume that $b$ is superficial for $(b, c) R$. Then $R$ is unmixed if and only if $R$ is a subspace of $D=A_{(M, b / c)}$.

Proof. By 4.5, $D$ is Macaulay, so the conclusion follows from 4.10 .

CoROLlaRY 4.13. With the notation of 4.1, assume that $b A: c A=(b A)^{(1)}$. Then $R$ is unmixed if and only if $R$ is a subspace of $D=A_{(M, b / c)}$.

Proof. By 4.7, $b$ is superficial for $(b, c) R$, so the conclusion follows from 4.12 .

COROLLARY 4.14. With the notation of 4.1, assume that $u \mathscr{R}(R,(b, c) R)$ has no height two prime divisors. Then $R$ is unmixed if and only if there exist $d \in(b, c) R$ and a prime ideal $P$ in $B=R[b / d, c / d]$ such that $M B \cong P$ and $R$ is a subspace of $B_{P}$.

Proof. $B$ is locally Macaulay 4.8, so the conclusion follows from 4.10 .

This section will be closed with the following remark which shows how the above results can be used to obtain information on when local domains of altitude $>2$ are unmixed.

REMARK 4.15. Let $(L, N)$ be a local domain such that altitude 
$L=n+1>2$, and let $x, y_{1}, \cdots, y_{n}$ be a system of parameters in $L$. Let $B=L\left[y_{1} / x, \cdots, y_{n} / x\right]$, and let $P=\left(M B, y_{1} / x\right) B$. Then $(R, M)=\left(B_{P}, P B_{P}\right)$ is a local domain of altitude two [9, Lemma 4.3]. Now, if $L$ is a subspace of $R$, then $L$ is unmixed if and only if $R$ is unmixed [9, Lemma 4.5(2)]. Therefore, assuming that $L$ is a subspace of $R, L$ is unmixed if and only if $L$ is a subspace of some Macaulay local domain of the form $A_{P}$, where $b, c \cdot$ is a system of parameters in $R$ and $P$ is a prime ideal in $A=R[b / c]$ such that $M A \subseteq P$.

Proof. This follows from [9, Lemma 4.5(2)] and 4.10.

Also, in 4.15 and assuming that $L$ is a subspace of $R, L$ is unmixed if and only if $R^{(1)}$ is a finite $R$-algebra, by [9, Lemma 4.5(2)] and 3.4.

5. Relationships between $R, R^{(1)}$, and $R^{\prime}$. In this section we consider what containment relationships are possible between $R, R^{(1)}$, and $R^{\prime}$, and what these imply concerning quasi-unmixedness, where $R$ is a local domain of altitude two and $R^{\prime}$ is the integral closure of $R$. 5.1 gives the main results, and 5.2 gives some supplemental information and some examples. Much of these two results is a compilation of known results.

TheOREM 5.1. Let $(R, M)$ be a local domain such that altitude $R=2$, and let $R^{\prime}$ be the integral closure of $R$. Let $\mathscr{P}=\{p \in \mathrm{Spec}$ $R$,; height $p=1\}$, so $R^{(1)}=\bigcap\left\{R_{p} ; p \in \mathscr{P}\right\}$. Then the following statements hold:

5.1.1. $R^{(1)} \supset R^{\prime}$ if and only if $R_{p}$ is integrally closed, for all $p \in \mathscr{P}$, and there exists a height one maximal ideal in $R^{\prime}$.

5.1.2. $R^{(1)}=R^{\prime}$ if and only if $R_{p}$ is integrally closed, for all $p \in \mathscr{P}$, and $R$ is quasi-unmixed.

5.1.3. $R^{(1)} \subseteq R^{\prime}$ if and only if $R$ is quasi-unmixed.

5.1.4. $R^{(1)}$ is a finite $R$-algebra if and only if $R$ is unmixed.

5.1.5. $R^{(1)}=R$ if and only if $R$ is Macaulay.

Proof. Let $\mathscr{P}^{\prime}=\left\{p^{\prime} \in \operatorname{Spec} R^{\prime}\right.$; height $\left.p^{\prime}=1\right\}$.

5.1.1. $R^{\prime} \leqq R^{(1)}$ if and only if $R^{\prime} \subseteq P_{p}$, for all $p \in \mathscr{P}$, if and only if $R_{p}=R_{(R-p)}^{\prime}$ is integrally closed, for all $p \in \mathscr{P}$, if and only if (by integral dependence) $R^{(1)}=\bigcap\left\{R_{p^{\prime}}^{\prime} ; p^{\prime} \in \mathscr{P}^{\prime \prime}\right\}$, where $\mathscr{P}^{\prime \prime}=\left\{p^{\prime} \in \mathscr{P}^{\prime} ; p^{\prime}\right.$ isn't maximal\}. Also, there exists a height one maximal ideal in $R^{\prime}$ if and only if $\mathscr{P}^{\prime \prime} \subset \mathscr{P}^{\prime}$ if and only if $R^{\prime}=[6,(2 . \mathrm{b}), \mathrm{p} .116] \cap$ $\left\{R_{p^{\prime}}^{\prime} ; p^{\prime} \in \mathscr{P}^{\prime}\right\} \subset \bigcap\left\{R_{p^{\prime}}^{\prime} ; p^{\prime} \in \mathscr{P}^{\prime \prime}\right\} . \quad 5.1 .1$ readily follows from this.

5.1.2. By 5.1.1, $R^{\prime}=R^{(1)}$ if and only if $R_{p}$ is integrally closed, 
for all $p \in \mathscr{P}$, and there does not exist a height one maximal ideal in $R^{\prime}$. 5.1.2 follows from this and the fact that there does not exist a height one maximal ideal in $R^{\prime}$ if and only if $R$ is quasi-unmixed, by [8, Corollary 3.4(i)].

5.1.3. $R$ is quasi-unmixed if and only if there does not exist a height one maximal ideal in $R^{\prime}$ [8, Corollary 3.4(i)] if and only if, for each $p^{\prime} \in \mathscr{P}^{\prime}, p^{\prime} \cap R \in \mathscr{P}$ if and only if $R^{(1)}=$ (by the Lying Over Theorem) $\cap\left\{R_{p^{\prime} \cap R} ; p^{\prime} \in \mathscr{P}^{\prime}\right\} \subseteq \bigcap\left\{R_{p^{\prime}}^{\prime} ; p^{\prime} \in \mathscr{P}^{\prime}\right\}=R^{\prime}$.

5.1.4. is given by 3.4.

5.1.5. $R$ is Macaulay if and only if, for each $0 \neq b \in M, b R$ is pure height one if and only if $R=R^{(1)}$ [10, Theorem 2.17].

REMARK 5.2. With the notation of 5.1 the following statements hold:

5.2.1. $R^{(1)} \subset R^{\prime}$ if and only if $R$ is quasi-unmixed and $R_{p}$ isn't integrally closed, for some $p \in \mathscr{P}$.

5.2.2. $R$ is quasi-unmixed if and only if height $M^{\prime}=2$, for all maximal ideals $M^{\prime}$ in $R^{\prime}$.

5.2.3. If $R^{\prime}=R$, then $R$ is Macaulay and $R=R^{(1)}$.

5.2.4. If $R^{(1)}$ is a finite $R$-algebra (so $R^{(1)} \subseteq R^{\prime}$ ), then either $R^{(1)}=R$ or the conductor $R: R^{(1)}$ of $R$ in $R^{(1)}$ is $M$-primary.

5.2.5. If $R^{\prime}$ is a finite $R^{(1)}$-algebra, then either $R^{(1)}=R^{\prime}$ or height $R^{(1)}: R^{\prime}=1$.

5.2.6. If $R$ is as in [2, Proposition 3.3], then $R^{(1)}=R^{\prime}$ and $R^{(1)}$ isn't a finite $R$-algebra. (Therefore $R$ is quasi-unmixed and isn't unmixed.)

5.2.7. If $R$ is as in [6, Example 2, pp. 203-205] in the case $m=0$, then $R^{(1)} \supset R^{\prime}$.

5.2.8. If $k$ is a field and $R=A_{P}$, where

$$
A=k\left[X^{2}-X, X^{3}-X^{2}, Y, X Y\right]
$$

and $P=\left(X^{2}-X, X^{3}-X^{2}, Y, X Y\right) A$, then $R^{(1)}=R^{\prime} \supset R$ and $R^{(1)}$ is a finite $R$-algebra.

5.2.9. There exists $x \in R^{\prime}$ such that $R^{(1)} \cong R^{\prime}[1 / x]=$ (say) $D^{\prime}, D^{\prime}$ is the integral closure of $D=R[x, 1 / x], D$ is a local domain, $D^{(1)}$ is a finite $R^{(1)}$-algebra, and the maximal ideals in $D^{\prime}$ are the ideals $M^{\prime} D^{\prime}$, where $M^{\prime}$ is a height two maximal ideal in $R^{\prime}$.

5.2.10. $R^{(1)}$ is Noetherian and is the smallest Macaulay domain $D$ such that: $R \cong D$, the integral closure $D^{\prime}$ of $D$ is $R^{\prime}[1 / x]$, for some $x \in R^{\prime}$, and all maximal ideals in $D$ have height two.

Proof. 5.2.1 is clear by 5.1.2 and 5.1.3.

5.2.2 follows from [8, Proposition 3.5].

5.2.3 holds, since an integrally closed local domain of altitude 
two is Macaulay.

5.2.4. Assume $(0) \neq R: R^{(1)} \neq R$, and let $0 \neq b \in M$. Then $\mathscr{T}(b R)=(3.5) R^{(1)}$ is a finite $R$-algebra, so $b^{n} \mathscr{T}(b R) \subseteq R$, for some $n \geqq 13.2 .5$. Therefore, since $M$ is finitely generated, $M^{k} R^{(1)} \subseteq R$, for some $k \geqq 1$, so $M^{k} \subseteq R: R^{(1)} \neq R$, hence $R: R^{(1)}$ is $M$-primary.

5.2.5. If $(0) \neq R^{(1)}: R^{\prime} \neq R^{\prime}$, then $R^{(1)} \subset R^{\prime}$, so there exists a height one prime ideal $p$ in $R$ such that $R^{\prime} \nsubseteq R_{p}$, hence $p^{*}=p R_{p} \cap R^{(1)}$ is a height one prime ideal such that $R^{\prime} \nsubseteq R_{p^{*}}^{(1)}$. Therefore

$$
(0) \neq R^{(1)}: R^{\prime} \subseteq p^{*},
$$

hence height $R^{(1)}: R^{\prime}=1$.

5.2.6 and 5.2.7 are given in the cited references. (The parenthetical statement in 5.2.6 follows from 5.1.3 and 5.1.4.)

5.2.8. The integral closure of $R$ is $R^{\prime}=k[X, Y]_{(X, Y) \cap(X-1, Y)}$ which is a regular domain with two maximal ideals, altitude $R=$ altitude $R^{\prime}=2$, and $R^{\prime}=R[X]$ is a finite $R$-algebra. Also, it is readily seen that, for each height one prime ideal $p$ in $R$, either $X$ or $1 / X$ is in $R_{p}$, and so in either case $X \in R_{p}$ (since $X \in R^{\prime}$ and $1 / X \in R_{p}$ imply $X \in R_{p}$ ). Therefore $R^{(1)} \supseteqq R^{\prime}$. Also, $R$ is quasi-unmixed, since $R$ is a locality over a field, so $R^{(1)}=R^{\prime}$ is a finite $R$-algebra 5.1.2.

5.2.9. If $R^{\prime}$ has no height one maximal ideals, then $R^{(1)} \subseteq R^{\prime}$, by 5.1.3 and 5.2.2, so let $x=1$. If $R^{\prime}$ has height one maximal ideals, then, since $R^{\prime}$ has only finitely many maximal ideals, let $x$ in all height one maximal ideals in $R^{\prime}$ such that $x-1$ is in all height two maximal ideals. Then $R^{\prime}[1 / x]$ is the integral closure of $D=R[x, 1 / x]$ and $D$ is a local domain of altitude two (since $R[x]$ has exactly two maximal ideals, say $N$ and $N^{\prime}$, height $N=1$, height $N^{\prime}=2, x \in N$, and $\left.x \notin N^{\prime}\right)$. Let $C=R+\left(N \cap N^{\prime}\right)$, so $R \subseteq C \leqq R[x]$. Also, $C$ is a local domain which is a finite $R$-algebra, so $C^{(1)}$ if a finite $R^{(1)}$-algebra (since height one prime ideals in $C$ contract to height one prime ideals in $R$ and, if $d \in R: C$, then $\left.d \in R^{(1)}: C^{(1)}\right)$. Finally, $D^{(1)}=C^{(1)}$ $[13,4.2]$, and the maximal ideals in $D^{\prime}$ are as described.

5.2.10. $R^{(1)}$ is Noetherian (by [2, Corollary 1.4]), every maximal ideal in the integral closure $R^{\prime}[1 / x]$ of $R^{(1)}$ has height two (by 5.2.9) (so every maximal ideal in $R^{(1)}$ has height two), and $b R^{(1)}$ is pure height one, for all nonzero nonunits $b \in R^{(1)}[3,5.10 .17(\mathrm{i})]$, so $R^{(1)}$ is Macaulay. Now let $D$ be a Macaulay domain such that $R \subseteq D \subseteq D^{\prime}=$ $R^{\prime}[1 / x]$, for some $x \in R^{\prime}$, and such that all maximal ideals in $D$ have height two. Let $p$ be a height one prime ideal in $D$. Then there exists a height one prime ideal $p^{\prime \prime}$ in $D^{\prime}=R^{\prime}[1 / x]$ such that $p^{\prime \prime} \cap D=p$ and $p^{\prime \prime}$ isn't maximal. Therefore $p^{\prime}=p^{\prime \prime} \cap R^{\prime}$ is a height one nonmaximal prime ideal, so $q=p^{\prime} \cap R$ is a height one prime ideal. Therefore $R_{q} \subseteq D_{p}$, hence $R^{(1)} \cong D^{(1)}=D$. 
In particular, by 5.2.10 and 5.1.3, if $R$ is quasi-unmixed, then $R^{(1)}$ is the smallest Macaulay domain $D$ such that $R \cong D \subseteq R^{\prime}$.

6. Some characterizations of arbitrary unmixed local domains. In this Section 6.1 gives a sufficient condition for a semi-local ring to be unmixed. Then 6.3-6.9 give some characterizations of unmixed local domains.

THEOREM 6.1. Let $R$ be a semi-local ring, and assume there exists a regular element $b$ in the Jacobson radical of $R$ such that $R / b R$ is unmixed. Then $R$ is unmixed.

Proof. Let $\mathscr{P}$ be the set of prime divisors of $b R^{*}$, where $R^{*}$ is the completion of $R$. Then, by hypothesis, each $p \in \mathscr{P}$ is such that depth $p=\operatorname{depth} b R^{*}=$ altitude $R-1$. Also, $b R^{*}=\bigcap\left\{b R_{p}^{*} \cap R^{*}\right.$; $p \in \mathscr{P}\} \supseteqq \bigcap\left\{(0) R_{p}^{*} \cap R^{*} ; p \in \mathscr{P}\right\}=$ (say) $N$. Then $N: b R=N$, since $b$ is a regular element in $R^{*}$, so $N=(0)[6,4.3]$. Therefore it follows that if $z$ is a prime divisor of $(0)$ in $R^{*}$, then there exists $p \in \mathscr{P}$ such that $z \subset p$. Then depth $z \geqq$ depth $p+1=$ altitude $R$, so $R$ is unmixed.

It is an open problem whether every quasi-unmixed local UFD $R$ is unmixed. (Note that if either $R$ is Macaulay or the completion of $R$ is a $U F D$, then $R$ is unmixed, but it is known that there exist local UFD's which aren't Macaulay and whose completions aren't UFD's.) Using 6.1, the following remark shows that if one such ring isn't unmixed, then many other examples (besides the one given in [1, Proposition 3.3]) of quasi-unmixed and not unmixed local domains exist.

REMARK 6.2. Let $R$ be a local domain such that altitude $R=a \geqq 3, R$ is quasi-unmixed, and $R$ is a UFD. If $R$ isn't unmixed, then, for all height one prime ideals $\pi R$ in $R, R / \pi R$ is a local domain, altitude $R / \pi R=a-1, R / \pi R$ is quasi-unmixed [6, 34.5], and $R / \pi R$ isn't unmixed 6.1.

It has recently been shown that if $R$ is an unmixed local domain, then, for all but finitely many height one prime ideals $p$ in $R, R / p$ is unmixed [1, 5.9] or [12, Remark 3.2(2)] together with [6, 18.11]. Using this, we have the following result which is related to 6.1.

Theorem 6.3. Let $(R, M)$ be a local domain, not a field. Then $R$ is unmixed if and only if there exists $0 \neq b \in M$ such that $\mathscr{T}(b R)$ is a finite $R$-algebra and $\mathscr{T} / b \mathscr{T}$ is unmixed. 
Proof. If $R$ is unmixed, then $R^{(1)}$ is a finite $R$-algebra [9, Lemma 5.11], so, for all $0 \neq b \in M, \mathscr{T}(b R)$ is a finite $R$-algebra. Also, there exists a finite (possibly empty) set $\mathscr{P}$ of height one prime ideals $p^{\prime}$ in $R$ such that $R / p^{\prime}$ isn't unmixed [1, 5.9] or [12, Remark 3.2(2)] together with $[6,18.11]$. Let $0 \neq b \in M$ such that $b \notin \bigcup\left\{p^{\prime} ; p^{\prime} \in \mathscr{P}\right\}$. (Since $R$ isn't a field, such $b$ exist.) Then $\mathscr{T}=$ $\mathscr{T}(b R)$ is a finite $R$-algebra, $b \mathscr{T}$ is pure height one 3.2.6, and there exists a one-to-one correspondence between the prime divisors $q$ of $b \mathscr{T}$ and the height one prime divisors $p$ of $b R$ such that $R_{p}=\mathscr{T}_{q}$ 3.2.1. Therefore, for each prime divisor $q$ of $b \mathscr{T}$ (and with $p=q \cap R$ ), $\mathscr{T} / q$ is unmixed (since $R / p$ is and $\mathscr{T} / q$ is a finite $R / p$-algebra with the same integral closure). Also, depth $q=$ altitude $R-1$, since $R$ is catenary $[6,34.6]$ and height $p=1$. Therefore $\mathscr{T} / b \mathscr{T}$ is unmixed, since a semi-local ring $\mathscr{T}$ is mixed if and only if $\mathscr{T} / z$ is unmixed and depth $z=$ altitude $\mathscr{T}$, for all prime divisors $z$ of zero in $\mathscr{T}$.

Conversely, $\mathscr{T}=\mathscr{T}(b R)$ is unmixed 6.1, so $R$ is unmixed, since, by hypothesis, the completions of $R$ and $\mathscr{T}$ have the same total quotient ring.

CoRollary 6.4. Let $(R, M)$ be as in 6.3. Then $R$ is unmixed if and only if there exists $0 \neq b \in M$ each that $\mathscr{T}(b R)$ is a finite $R$-algebra and, for each minimal prime divisor $p$ of $b R$, depth $p=$ altitude $R-1$ and $R / p$ is unmixed.

Proof. If $R$ is unmixed, then there exists $0 \neq b \in M$ such that $\mathscr{T}=\mathscr{T}(b R)$ is a finite $R$-algebra and $\mathscr{T} / b \mathscr{T}$ is unmixed 6.3. Therefore, by the one-to-one correspondence between the prime divisors $q$ of $b \mathscr{T}$ and the height one prime divisors $p$ of $b R$ 3.2.1, for each minimal prime divisor $p$ of $b R$, depth $p=$ altitude $R-1$ and $R / p$ is unmixed (since $\mathscr{T} / q$ is a finite $R / p$-algebra with the same integral closure).

Conversely, it suffices to prove that $\mathscr{T}=\mathscr{T}(b R)$ is such that $\mathscr{T} / b \mathscr{T}$ is unmixed, by 6.3. But this follows from the hypothesis and the one-to-one correspondence mentioned above, since $b \mathscr{T}$ is pure height one 3.2.6.

REMARK 6.5. The proofs of 6.3 and 6.4 show that if $R$ is unmixed, then for each $b \in M, \notin \mathbf{U}\{q \in \operatorname{Spec} R$; height $q=1$ and $R / q$ isn't unmixed (which is a finite set $[1,5.9], \mathscr{T}=\mathscr{T}(b R)$ is a finite $R$-algebra and $\mathscr{T} / b \mathscr{T}$ is unmixed (in (6.3) and $R / p$ is unmixed, for all minimal prime divisors $p$ of $b R$ (in 6.4)).

There is a somewhat analogous characterization of a quasiunmixed local domain, as will now be shown. The author doesn't 
know if the quantifier in the condition in 6.6 can be replaced by "there exists." (However, if it is assumed that $R / p$ is unmixed (rather than quasi-unmixed), for all height one prime ideals $p$ in $R$, then this replacement can be made.)

Proposition 6.6. Let $(R, M)$ be a local domain. Then $R$ is quasi-unmixed if and only if, for all $0 \neq b \in M, \mathscr{T}(b R) \subseteq R^{\prime}$ (=the integral closure of $R$ ) and, for each minimal prime divisor $p$ of $b R$, depth $p=$ altitude $R-1$ and $R / p$ is quasi-unmixed.

Proof. If $R$ is quasi-unmixed, then $R^{(1)} \subseteq R^{\prime}$ [9, Theorem 5.4], so, for all $0 \neq b \in M, \mathscr{T}(b R) \subseteq R^{\prime}$. Also, for each height one prime ideal $p$ in $R$, depth $p=$ altitude $R-1$ and $R / p$ is quasi-unmixed $[6,34.5]$.

For the converse, it may clearly be assumed that altitude $R>1$. Let $z$ be a minimal prime ideal in the completion $R^{*}$ of $R$. Then, since $\mathscr{T}(b R) \subseteq R^{\prime}$, for each $0 \neq b \in M$, there are no height one maximal ideals in $R^{\prime}$ [15, Corollary 2.13], so depth $z>1$ [8, Proposition 3.5]. Also, there are at most a finite number of prime ideals $q$ in $R^{*}$ such that $z \subset q$ and height $q / z=1<$ height $q[4$, Theorem 1]. Let $\mathscr{P}$ be this set of prime ideals, so $M R^{*} \notin \mathscr{P}$. Therefore let $0 \neq b \in M$ such that $b \notin \bigcup\{q \cap R ; q \in \mathscr{P}\}$. Then there exists a prime ideal $p^{*}$ in $R^{*}$ such that $(z, b) R^{*} \subseteq p^{*}$ and height $p^{*} / z=1$, so height $p^{*}=1$ (by the choice of $b$ ). Therefore $p^{*}$ is a minimal prime divisor of $b R^{*}$, so $p^{*}$ is a minimal prime divisor of $p R^{*}$, where $p=p^{*} \cap R$. Then height $p=1$, so by hypothesis, depth $p^{*}=$ depth $p=$ altitude $R-1$, hence depth $z=$ altitude $R$, so $R$ is quasi-unmixed.

It is known [9, Theorem 5.3] that for a local domain $R, R$ is catenary and $(R / p)^{(1)}$ is a finite $R / p$-algebra, for all $p \in \operatorname{Spec} R$ if and only if $R / p$ is unmixed, for all $p \in \operatorname{Spec} R$. The following result shows that a weaker condition than that in [9, Theorem 5.3] implies that $R / p$ is unmixed, for all $p \in \operatorname{Spec} R$.

THeOREM 6.7. Let $(R, M)$ be a local domain. Then, for all $p \in \operatorname{Spec} R, R / p$ is unmixed if and only if the following condition holds: $R$ is catenary and, for all non-maximal $p \in \operatorname{Spec} R$, there exists $0 \neq b+p \in M / p$ such that $\mathscr{T}((b+p)(R / p))$ is a finite $R / p$ algebra.

Proof. Assume first that the condition holds. The proof that each $R / p$ is unmixed is by induction on $n=$ altitude $R$, and it may clearly be assumed that $n>1$. Also, the condition is inherited by 
each $R / p$, so it suffices to prove that $R$ is unmixed. For this, with $b$ as in the condition for $p=(0), \mathscr{T}(b R)$ is a finite $R$-algebra. Also, for each minimal prime divisor $p$ of $b R, R / p$ is unmixed (by induction). Therefore, since $R$ is catenary, $R$ is unmixed 6.4.

The converse follows immediately from [9, Theorem 5.3], since $\mathscr{T}((b+p)(R / p)) \subseteq(R / p)^{(1)}$.

The following result shows that a somewhat analogous condition to that in 6.7 characterizes when $R$ is unmixed.

Theorem 6.8. Let $(R, M)$ be a local ring such that $n=$ altitude $R>0$ and all prime divisors of zero are minimal. Then $R$ is unmixed if and only if $R$ satisfies the first chain condition (f.c.c.) and there exists a system of parameters (s.o.p.) $x_{1}, \cdots, x_{n}$ in $R$ such that the following condition holds for $i=0,1, \cdots, n-2$ : if $q$ is a minimal prime divisor of $\left(x_{1}, \cdots, x_{i}\right) R$, then $\mathscr{T}\left(\left(x_{i+1}+q\right)(R / q)\right)$ is a finite $R / q$-algebra. (For $i=0, q$ is a minimal prime divisor of zero.) Moreover, if $R$ is unmixed, then, for each $q \in \mathscr{P}=\{q \in \operatorname{Spec} R ; q$ is a minimal prime divisor of $\left(x_{1}, \cdots, x_{i}\right) R$, for some $\left.i=0,1, \cdots, n\right\}$, $R / q$ is unmixed.

Proof. Assume first that $R$ is unmixed, so $R$ satisfies the f.c.c. $[6,34.6]$. Let $z_{1}, \cdots, z_{g}$ be the (minimal) prime divisors of zero in $R$. The existence of $x_{1}, \cdots, x_{n}$ will be shown by induction on $n$. If $n=1$, then there is nothing to prove, so assume that $n>1$ and the result holds for local rings of altitude $n-1$. For each $i=1, \cdots, g, \quad U_{i}=\left\{p \in \operatorname{Spec} R ; z_{i} \subset p\right.$, height $p / z_{i}=1$, and $R / p$ isn't unmixed $\}$ is a finite set $[1,5.9]$. Therefore let $x_{1} \in M, \notin \bigcup\left\{p ; p \in \bigcup U_{i}\right\}$. Now, for $i=1, \cdots, g, R / z_{i}$ is unmixed [5, Proposition 3], so $\mathscr{T}\left(\left(x_{1}+z_{i}\right)\left(R / z_{i}\right)\right)$ is a finite $R / z_{i}$-algebra 6.5. Also, for each minimal prime divisor $Q$ of $\left(x_{1}+z_{i}\right)\left(R / z_{i}\right),\left(R / z_{i}\right) / Q$ is unmixed (by the choice of $b$ ) and depth $Q=\operatorname{depth} z_{i}-1=n-1$. Let $Z=\bigcap\{P \in \operatorname{Spec} R$; $P$ is a minimal prime divisor of $x_{1} R$. Then $R / Z$ is unmixed [5, Proposition 3] (since each $(R / Z) /(P / Z)$ is unmixed and altitude $R / P=\operatorname{depth} P / Z=n-1$ (since $R$ satisfies the f.c.c.)), so by induction, let $x_{2}, \cdots, x_{n} \in M$ such that their residue classes modulo $Z$ are a s.o.p. in $R / Z$ that satisfy the condition. Then it readily follows that $x_{1}, \cdots, x_{n}$ is a s.o.p. in $R$ which satisfy the condition.

Conversely, assume that $R$ satisfies the f.c.c. and has a s.o.p. which satisfy the condition. Then it will be shown by induction on $n$ that $R$ is unmixed. If $n=1$, then it is well known that $R$ is unmixed (since all prime divisors of zero are minimal), so assume that $n>1$. Let $z_{1}, \cdots, z_{g}$ be the (minimal) prime divisors of zero in $R$, and fix $z=z_{i}$. Now, by hypothesis, $\mathscr{T}\left(\left(x_{1}+z\right)(R / z)\right)$ is a finite 
$R / z$-algebra and, for each minimal prime divisor $Q$ of $\left(x_{1}+z\right)(R / z)$, depth $Q=$ altitude $R / z-1=n-1$. Further, $(R / z) / Q$ is unmixed (by induction on $n$, since $(R / z) / Q$ satisfies the f.c.c. and the residue classes of $x_{2}, \cdots, x_{n}$ are a s.o.p. which satisfy the condition). Therefore $R / z$ is unmixed 6.4 and depth $z=$ altitude $R$ (by the f.c.c.), so $R$ is unmixed [5, Proposition 3].

Finally, let $R$ be unmixed and $q \in \mathscr{P}$, say $q$ is a minimal prime divisor of $\left(x_{1}, \cdots, x_{i}\right) R$. Then $R / q$ satisfies the f.c.c. and the residue classes modulo $q$ of $x_{i+1}, \cdots, x_{n}$ are a s.o.p. which satisfy the condition, so $R / q$ is unmixed (by what has already been proved, for $0 \leqq i \leqq n-2$; and clearly for $i=n-1, n)$.

The final result in this paper characterizes in an unmixed local domain $R$ when $R /(\operatorname{Rad} b R)$ is unmixed. (This result is of some interest, since it is still an open problem whether $R / p$ is unmixed, for all height one prime ideals $p$ in an unmixed local domain $R$.) For this characterization, we extend the definition of $\mathscr{T}(b A)$ to the case of a Noetherian ring (with non-zero divisors of zero) as follows: if $b$ is a regular element in a Noetherian ring $A$, then $\mathscr{T}(b A)=$ $A[1 / b] \cap A_{[S]}$, where $S=A-\cup\{p \in \operatorname{Spec} A ; b \in p$ and height $p=1\}$ and $A_{[S]}=\{a / s ; a \in A$ and $s$ is a regular element in $S\}$. (If $p \in \operatorname{Spec} A$, then let $A_{[p]}=A_{[A-p]}$.) (Most of the statements in 3.2 continue to hold in this case, as is proved in the cited references.)

THEOREM 6.9. Let $R$ be an unmixed semi-local domain, and let $0 \neq b \in J=$ the Jacobson radical of $R$. Then $R /(\operatorname{Rad} b R)$ is unmixed if and only if the completion of $\mathscr{T}(b R)$ is $\mathscr{T}\left(b R^{*}\right)$, where $R^{*}$ is the completion of $R$.

Proof. Since $R$ is unmixed, $R^{(1)}$ is a finite $R$-algebra [9, Lemma 5.11], so $\mathscr{T}(b R)$ is a finite $R$-algebra, for each $0 \neq b \in J$. Fix $0 \neq b \in J$, and let $\mathscr{T}^{*}$ be the completion of $\mathscr{T}=\mathscr{T}(b R)$. Then, as will now be shown, $\mathscr{T}^{*} \subseteq \mathscr{T}\left(b R^{*}\right)$ and $\mathscr{T}\left(b R^{*}\right) \cap F=\mathscr{T}$, where $F$ is the quotient field of $R$. Namely, it is readily seen that $R^{*}[1 / b] \cap F=R[1 / b]$. Also, for each minimal prime divisor $p^{*}$ of $b R^{*}, R_{p^{*}}^{*}$ is a faithfully flat $R_{p}$-algebra $[6,19.2(3)]$, where $p=p^{*} \cap R$ (so $p$ is a minimal prime divisor of $b R$ ). Therefore $R_{p^{*}}^{*} \cap F=R_{p}[6$, 18.4], so it readily follows that $R_{\left[p^{*}\right]}^{*} \cap F=R_{p}$. Further, if $q$ is a minimal prime divisor of $b R$, then there exists a minimal prime divisor $q^{*}$ of $b R^{*}$ such that $q^{*} \cap R=q[6,18.11]$, hence $R_{\left[q^{*}\right]}^{*} \cap F=R_{q}$. Therefore $\mathscr{T}\left(b R^{*}\right) \cap F=\mathscr{T}(b R)$, and so $\mathscr{T}^{*}=R^{*}[\mathscr{T}] \subseteq \mathscr{T}\left(b R^{*}\right)$.

Now $b \mathscr{T}\left(b R^{*}\right)$ is pure height one 3.2.6, and $\mathscr{T}\left(b R^{*}\right)$ is the smallest ring $A$ such that $R^{*} \subseteq A \subseteq R^{* \prime}$ (=the integral closure of $R^{*}$ ) such that $b A$ is pure height one. For, if $b A$ is pure height 
one, then, with $S^{\prime}=\{p \in \operatorname{Spec} A ; b \in p$ and height $p=1\}$ and $U^{\prime}=$ $A-\bigcup\left\{p ; p \in S^{\prime}\right\}, A=A[1 / b] \cap A_{\left[U^{\prime}\right]}$ (since if $x \in A[1 / b] \cap A_{\left[U^{\prime}\right]}$, then $x=a / b^{k}$, for some $a \in A$ and $k \geqq 1$, and $a \in b^{k} A_{\left[U^{\prime}\right]} \cap A=b^{k} A$, so $\dot{x} \in A$; and the opposite conclusion is clear). Also, if $p \in S^{\prime}$, then $p \cap R^{*}$ is a height one prime divisor of $b R^{*}$ (since $R$ is unmixed implies that height $p \cap R^{*}=$ height $p$ ). Therefore, with $S=\left\{p^{*} \epsilon\right.$ Spec $R^{*} ; b \in p^{*}$ and height $\left.p^{*}=1\right\}, \mathscr{T}\left(b R^{*}\right)=R^{*}[1 / b] \cap R_{[U]}^{*} \cong A$, where $U=R^{*}-\bigcup\left\{p^{*} ; p^{*} \in S\right\}$.

Therefore, since $\mathscr{T}^{*} \subseteq \mathscr{T}\left(b R^{*}\right), b \mathscr{T}^{*}$ is pure height one if and only if $\mathscr{T}^{*}=\mathscr{T}\left(b R^{*}\right)$. Also, since $\mathscr{T}$ is unmixed (since $R$ is), $b \mathscr{T}^{*}$ is pure height one if and only if every prime divisor of $b \mathscr{T}^{*}$ has depth = altitude $R-1$ if and only if $\mathscr{T} / b \mathscr{T}$ is unmixed if and only if $R /(\operatorname{Rad} b R)$ is unmixed (as in the first paragraph of the proof of 6.4, since a semi-local ring $L$ is unmixed if and only if, for each prime divisor $z$ of zero in $L$, depth $z=$ altitude $L$ and $L / z$ is unmixed).

\section{REFERENCES}

1. M. Brodmann, Über die minimale dimension der assoziierten primideale der kompletion eines lokalen integritaetsbereiches, Ph. D. Dissertation, Universität Basel, Basel, Switzerland, 1974.

2. D. Ferrand and M. Raynaud, Fibres formelles d'un anneau local Noethérian, Ann. Sci. Ecole Norm. Sup. 3 (1970), 295-311.

3. A. Grothendieck, Éléments de Géométrie Algebrique IV (Seconde Partie), Institut de Hautes Etudes Scientifiques, Paris, 1964.

4. S. McAdam, Saturated chains in Noetherian rings, Indiana Univ. Math. J., 23 (1974), 719-728.

5. M. Nagata, On the chain problem of prime ideals, Nagoya Math. J., 10 (1956), $51-64$.

6. — Local Rings, Interscience Tracts 13, Interscience, New York, 1962.

7. L. J. Ratliff, Jr., Two notes on locally Macaulay rings, Trans. Amer. Math. Soc., 119 (1965), 399-406.

8. - On quasi-unmixed local domains, the altitude formula, and the chain condition for prime ideals (I), Amer. J. Math., 91 (1969), 508-528.

9. - On quasi-unmixed local domains, the altitude formula, and the chain condition for prime ideals (II), Amer. J. Math., 92 (1970), 99-144.

10. - On prime divisors of the integral closure of a principal ideal, J. Reine Angew. Math., 255 (1972), 210-220.

11. Conditions for $\operatorname{Ker}(R[X] \rightarrow R[c / b])$ to have a linear base, Proc. Amer. Math. Soc., 39 (1973), 509-514.

12. Three theorems on imbedded prime divisors of principal ideals, Pacific J. Math., 49 (1973), 199-210.

13. - Four notes on saturated chains of prime ideals, J. Algebra, 39 (1976), 75-93.

14. D. Rees, Valuations associated with ideals, Proc. London Math. Soc., 3 (1956), 161-174.

15. P. G. Sawtelle, Characterizations of unmixed and quasi-unmixed local domains, Ph. D. Dissertation, University of Calif., Riverside, CA, 1971.

16. V. M. Smith, Strongly superficial elements, Pacific J. Math., 58 (1975), 643-650. 
17. O. Zariski and P. Samuel, Commutative Algebra, Vol. 2, D. van Nostrand Co., Inc., New York, NY, 1960.

Received November 26, 1975 . Research on this paper was supported in part by the National Science Foundation Grant MPS71-02929 A03.

University of CALifornia, Riverside 


\section{PACIFIC JOURNAL OF MATHEMATICS}

\section{EDITORS}

RICHARD ARENS (Managing Editor)

University of California

Los Angeles, California 90024

\section{R. A. Beaumont}

University of Washington

Seattle, Washington 98105
J. DugundjI

Department of Mathematics

University of Southern California

Los Angeles, California 90007

D. Gilbarg and J. Milgram

Stanford University

Stanford, California 94305

\section{ASSOCIATE EDITORS}
E. F. BECKENBACH
B. H. NeumanN
F. WOLF
K. YosHIDA

\section{SUPPORTING INSTITUTIONS}

\author{
UNIVERSITY OF BRITISH COLUMBIA \\ CALIFORNIA INSTITUTE OF TECHNOLOGY \\ UNIVERSITY OF CALIFORNIA \\ MONTANA STATE UNIVERSITY \\ UNIVERSITY OF NEVADA \\ NEW MEXICO STATE UNIVERSITY \\ OREGON STATE UNIVERSITY \\ UNIVERSITY OF OREGON \\ OSAKA UNIVERSITY
}

\author{
UNIVERSITY OF SOUTHERN CALIFORNIA \\ STANFORD UNIVERSITY \\ UNIVERSITY OF HAWAII \\ UNIVERSITY OF TOKYO \\ UNIVERSITY OF UTAH \\ WASHINGTON STATE UNIVERSITY \\ UNIVERSITY OF WASHINGTON \\ AMERICAN MATHEMATICAL SOCIETY
}

The Supporting Institutions listed above contribute to the cost of publication of this Journal, but they are not owners or publishers and have no responsibility for its content or policies.

Mathematical papers intended for publication in the Pacific Journal of Mathematics should be in typed form or offset-reproduced, (not dittoed), double spaced with large margins. Please do not use built up fractions in the text of your manuscript. You may however, use them in the displayed equations. Underline Greek letters in red, German in green, and script in blue. The first paragraph or two must be capable of being used separately as a synopsis of the entire paper. Items of the bibliography should not be cited there unless absolutely necessary, in which case they must be identified by author and Journal, rather than by item number. Manuscripts, in triplicate, may be sent to any one of the editors. Please classify according to the scheme of Math. Reviews, Index to Vol. 39. All other communications should be addressed to the managing editor, or Elaine Barth, University of California, Los Angeles, California, 90024.

The Pacific Journal of Mathematics expects the author's institution to pay page charges, and reserves the right to delay publication for nonpayment of charges in case of financial emergency.

100 reprints are provided free for each article, only if page charges have been substantially paid. Additional copies may be obtained at cost in multiples of 50 .

The Pacific Journal of Mathematics is issued monthly as of January 1966. Regular subscription rate: $\$ 72.00$ a year (6 Vols., 12 issues). Special rate: $\$ 36.00$ a year to individual members of supporting institutions.

Subscriptions, orders for back numbers, and changes of address should be sent to Pacific Journal of Mathematics, 103 Highland Boulevard, Berkeley, California, 94708.

PUBLISHED BY PACIFIC JOURNAL OF MATHEMATICS, A NON-PROFIT CORPORATION

Printed at Kokusai Bunken Insatsusha (International Academic Printing Co., Ltd.), 8-8, 3-chome, Takadanobaba, Shinjuku-ku, Tokyo 160, Japan. 


\section{Pacific Journal of Mathematics \\ Vol. 65, No. $2 \quad$ October, 1976}

Andrew Adler, Weak homomorphisms and invariants: an example .......... 293

Howard Anton and William J. Pervin, Separation axioms and metric-like

functions ............................................. 299

Ron C. Blei, Sidon partitions and p-Sidon sets .................... 307

T. J. Cheatham and J. R. Smith, Regular and semisimple modules ........... 315

Charles Edward Cleaver, Packing spheres in Orlicz spaces .............. 325

Le Baron O. Ferguson and Michael D. Rusk, Korovkin sets for an operator on a

space of continuous functions ............................. 337

Rudolf Fritsch, An approximation theorem for maps into Kan fibrations ....... 347

David Sexton Gilliam, Geometry and the Radon-Nikodym theorem in strict

Mackey convergence spaces .................................

William Hery, Maximal ideals in algebras of topological algebra valued

functions ...................................... 365

Alan Hopenwasser, The radical of a reflexive operator algebra ........... 375

Bruno Kramm, A characterization of Riemann algebras................. 393

Peter K. F. Kuhfittig, Fixed points of locally contractive and nonexpansive

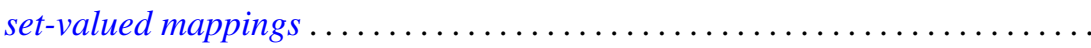

Stephen Allan McGrath, On almost everywhere convergence of Abel means of

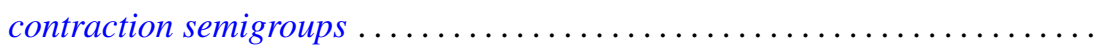

Edward Peter Merkes and Marion Wetzel, A geometric characterization of

indeterminate moment sequences............................ 409

John C. Morgan, II, The absolute Baire property ................... 421

Eli Aaron Passow and John A. Roulier, Negative theorems on generalized convex approximation .................................... 437

Louis Jackson Ratliff, Jr., A theorem on prime divisors of zero and characterizations of unmixed local domains ..............

Ellen Elizabeth Reed, A class of $T_{1}$-compactifications................... 471

Maxwell Alexander Rosenlicht, On Liouville's theory of elementary

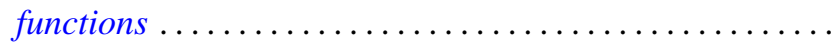

Arthur Argyle Sagle, Power-associative algebras and Riemannian

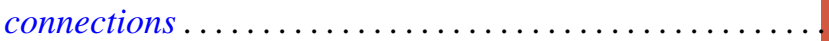

Chester Cornelius Seabury, On extending regular holomorphic maps from Stein manifolds...

Elias Sai Wan Shiu, Commutators and numerical ranges of powers of operators ...................................

Donald Mark Topkis, The structure of sublattices of the product of $n$ lattices ... 525

John Bason Wagoner, Delooping the continuous $K$-theory of a valuation

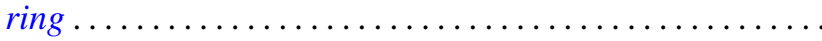

Ronson Joseph Warne, Standard regular semigroups...........

Anthony William Wickstead, The centraliser of $E \otimes_{\lambda} F \ldots$. 